\title{
Spontaneous Glial Calcium Waves in the Retina Develop over Early Adulthood
}

\author{
Zeb L. Kurth-Nelson, Anusha Mishra, and Eric A. Newman \\ Department of Neuroscience, University of Minnesota, Minneapolis, Minnesota 55455
}

Intercellular glial $\mathrm{Ca}^{2+}$ waves constitute a signaling pathway between glial cells. Artificial stimuli have previously been used to evoke these waves, and their physiological significance has been questioned. We report here that $\mathrm{Ca}^{2+}$ waves occur spontaneously in rat retinal glial cells, both in the isolated retina and in vivo. These spontaneous waves are propagated by ATP release. In the isolated retina, suramin (P2 receptor antagonist) reduces the frequency of spontaneous wave generation by $53 \%$, and apyrase (ATP-hydrolyzing enzyme) reduces frequency by $95-100 \%$. Luciferin-luciferase chemiluminescence reveals waves of ATP matching the spontaneous $\mathrm{Ca}^{2+}$ waves, indicating that ATP release occurs as spontaneous $\mathrm{Ca}^{2+}$ waves are generated. Wave generation also depends on age. Spontaneous wave frequency rises from 0.27 to 1.0 per minute per $\mathrm{mm}^{2}$, as rats age from 20 to $120 \mathrm{~d}$. The sensitivity of glia to ATP does not increase with age, but the ATP released by evoked waves is $31 \%$ greater in 120-d-old than in 20-d-old rats, suggesting that increased ATP release in older animals could account for the higher frequency of wave generation. Simultaneous imaging of glial $\mathrm{Ca}^{2+}$ and arterioles in the isolated retina demonstrates that spontaneous waves alter vessel diameter, implying that spontaneous waves may have a significant impact on retinal physiology. Spontaneous intercellular glial $\mathrm{Ca}^{2+}$ waves also occur in the retina in vivo, with frequency, speed, and diameter similar to the isolated retina. Increased spontaneous wave occurrence with age suggests that wave generation may be related to retinal pathology.

\section{Introduction}

Glial cells exhibit intracellular $\mathrm{Ca}^{2+}$ elevations in response to signals from neurons and other glia. Activation of metabotropic receptors on glia by neurotransmitters evokes release of $\mathrm{Ca}^{2+}$ from internal stores into the cytosol (Fellin and Carmignoto, 2004). This $\mathrm{Ca}^{2+}$ elevation can lead to release of glutamate, ATP and D-serine, altering synaptic efficacy (Araque et al., 1998; Kang et al., 1998; Yang et al., 2003; Pascual et al., 2005) and neuronal excitability (Parpura et al., 1994; Newman, 2003; Fellin et al., 2004). Glial $\mathrm{Ca}^{2+}$ elevations also trigger release of arachidonic acid metabolites that regulate blood vessel diameter (Zonta et al., 2003; Mulligan and MacVicar, 2004; Metea and Newman, 2006; Gordon et al., 2008).

Glia also exhibit spontaneous increases in intracellular $\mathrm{Ca}^{2+}$. Spontaneous $\mathrm{Ca}^{2+}$ transients in individual astrocytes were first reported in vitro (Cornell-Bell et al., 1990) and are also observed in situ (Aguado et al., 2002; Nett et al., 2002), where they can induce neuronal NMDAR currents (Parri et al., 2001). Spontaneous $\mathrm{Ca}^{2+}$ transients also occur in vivo and may regulate neurite growth and cause dilation of blood vessels (Takano et al., 2006; Kanemaru et al., 2007). These spontaneous transients remain confined to individual cells, although they may be spatially coordinated (Hirase et al., 2004).

\footnotetext{
Received May 28, 2009; revised July 27, 2009; accepted Aug. 9, 2009.

This work was supported by National Institutes of Health Grant EY004077 and NIH Vision Training Grant. We thank Janet Dubinsky, Benjamin Clark, and Anja Srienc for helpful discussions and comments on this manuscript, and Michael Burian for technical assistance.

Correspondence should be addressed to Eric A. Newman, Department of Neuroscience, University of Minnesota,

6-145 Jackson Hall, 321 Church Street SE, Minneapolis, MN 55455. E-mail: ean@umn.edu. DOI:10.1523/JNEUROSCI.2493-09.2009

Copyright $\odot 2009$ Society for Neuroscience 0270-6474/09/2911339-08\$15.00/0
}

When triggered by an external stimulus, calcium elevations can travel between glial cells in the form of propagated waves (Scemes and Giaume, 2006), coordinating glial activity over tens or hundreds of micrometers. These intercellular glial $\mathrm{Ca}^{2+}$ waves can be evoked by electrical, chemical, and mechanical stimuli and have been observed in astrocyte cultures (Cornell-Bell et al., 1990), the isolated retina (Newman and Zahs, 1997), brain slices (Kang et al., 1998; Schipke et al., 2002; Haas et al., 2006), and in vivo in the cortex (Tian et al., 2006; Ding et al., 2007). Depending on the preparation, evoked waves may propagate by release of ATP into the extracellular space, by diffusion of an intracellular messenger through the gap junctions between glial cells, or both (Scemes and Giaume, 2006). Evoked waves can modulate neuronal activity (Nedergaard, 1994; Newman and Zahs, 1998) and blood flow (Mulligan and MacVicar, 2004; Metea and Newman, 2006).

Recently, intercellular glial $\mathrm{Ca}^{2+}$ waves have been observed to occur spontaneously in vivo (Hoogland et al., 2009; Kuchibhotla et al., 2009), answering the important question of whether these waves occur under physiological conditions in the absence of artificial stimulation. We report here that spontaneous glial $\mathrm{Ca}^{2+}$ waves also occur in the retina, both in the isolated retina and in vivo. Like evoked waves, spontaneous waves in the retina are propagated by the release of ATP and can modulate blood vessel diameter. We also find that spontaneous waves increase in frequency as animals age. The results demonstrate that glia can independently regulate blood flow and suggest that glial $\mathrm{Ca}^{2+}$ waves may be involved in the aging process.

\section{Materials and Methods}

Animals. Male Long-Evans rats were used in all experiments. Rats were $20-100$-d-old on arrival from the supplier (Harlan Laboratories). Some 
were maintained for as long as an additional 9 months in the University of Minnesota Research Animal Resources facilities. Rats were killed by cutting the diaphragm following deep isoflurane anesthesia. The animals used in this study were treated in accordance with the guidelines of the Institutional Animal Care and Use Committee of the University of Minnesota.

Isolated retina preparation. The isolated retina preparation has been described previously (Newman, 2001). Briefly, retinas were dissected from the hemisected eyeball and the vitreous removed. Retinas were cut into two pieces and laid flat in a superfusion chamber with the vitreal surface up. Isolated retinas were superfused with bicarbonate-buffered Ringer's at $2-3 \mathrm{ml} / \mathrm{min}$ at $22-23^{\circ} \mathrm{C}$. All experiments except those described in Figure 7 were performed in the isolated retina.

Calcium and ATP imaging in the isolated retina. Our $\mathrm{Ca}^{2+}$ and ATP imaging methods have been described previously (Newman, 2001). For confocal $\mathrm{Ca}^{2+}$ imaging, retinas were incubated in the $\mathrm{Ca}^{2+}$ indicator dye Fluo-4 AM (25 $\mu \mathrm{g} / \mathrm{ml})$ and pluronic acid $(1.75 \mathrm{mg} / \mathrm{ml})$ for 25 min before imaging. Fluo-4 AM loaded selectively into glial cells and not neurons, consistent with previous reports (Porter and McCarthy, 1996; Stosiek et al., 2003; Hirase et al., 2004). Fluorescence images were captured with a confocal scanner (Odyssey, Noran), with images averaged for one second.

The luciferin-luciferase chemiluminescence assay was used to image ATP. Luciferase catalyzes the reaction of luciferin with ATP to emit light, allowing visualization of ATP release into the extracellular space. Superfusion was stopped and a solution of luciferin and luciferase gently pipetted into the chamber yielding final concentrations of $0.68 \mathrm{mg} / \mathrm{ml}$ luciferin and $0.61 \mathrm{mg} / \mathrm{ml}$ luciferase when imaging evoked ATP waves. Luciferase concentration was reduced by two thirds when imaging spontaneous waves because we found that luciferase itself acted as an inhibitor of spontaneous waves, presumably through its extracellular ATPase activity. The chemiluminescence was captured with a KS-1381 image intensifier (VideoScope) coupled to a CoolSnap ES camera (Photometrics) using a $1 \mathrm{~s}$ integration time with $2 \times 2$ pixel binning. To maintain $\mathrm{pH}$ and oxygenation during ATP imaging, a collar was fitted around the objective and superfusion chamber and $95 \% \mathrm{O}_{2}, 5 \% \mathrm{CO}_{2}$ gently blown over the surface of the chamber. All image acquisition was in MetaMorph (Molecular Devices). Luciferin-luciferase chemiluminescence images were calibrated as previously described (Newman, 2001) to calculate absolute ATP concentration at the retinal surface.

Calcium wave imaging and analysis. A $10 \times$ water-immersion objective (Olympus) was used to count waves in the isolated retina, covering an area of $\sim 0.5 \mathrm{~mm}^{2}$. Waves were tallied by the number of waves starting in each frame. Waves were distinguished from other time-varying fluorescence signals by their pattern of concentric expansion and mesh-like appearance (ganglion cells, which show little to no fluorescence signal, form the dark spaces in the mesh). A custom image analysis program was written to automatically detect and tally waves.

To simultaneously monitor blood vessel diameter and glial $\mathrm{Ca}^{2+}$, blood vessels were filled with dextran-conjugated fluorescein $(2,000,000$ MW; excites and emits at wavelengths similar to Fluo-4) by jugular vein injection before animal death to visualize the vessel lumen. In these experiments, retinas were incubated and superfused with $100 \mathrm{nM} \mathrm{U}-46619$ (an analog of thromboxane) to increase basal vessel tone (Metea and Newman, 2006).

In apyrase experiments, superfusion was stopped and apyrase gently pipetted into the recording chamber. In control experiments for apyrase, superfusion was likewise stopped and an equal volume of Ringer's pipet-
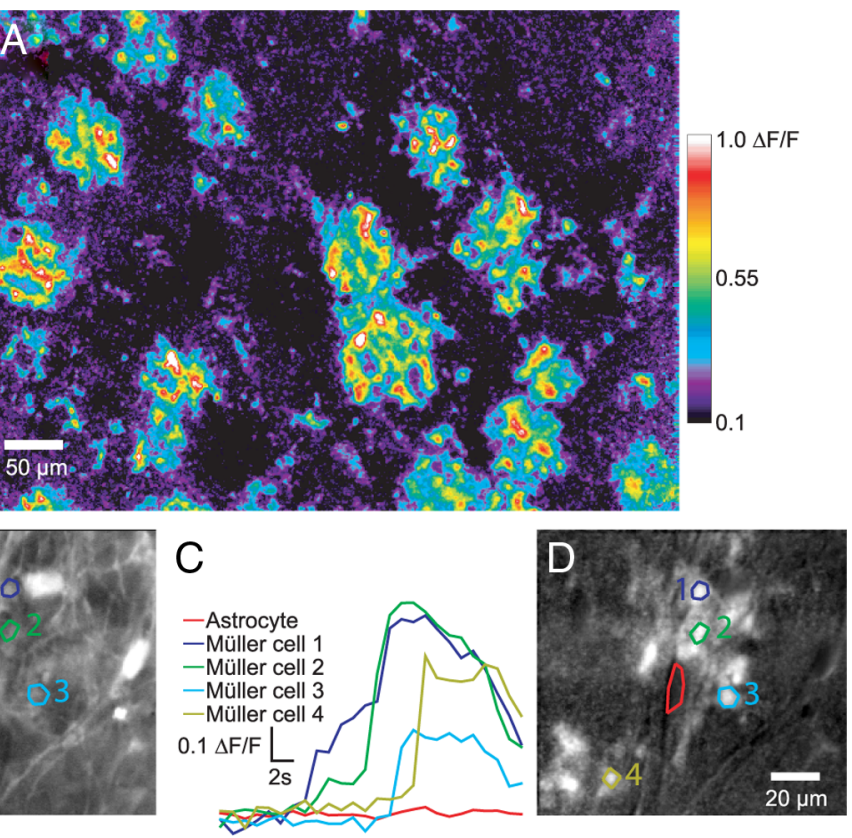

Figure 1. Spontaneous intercellular glial $\mathrm{Ca}^{2+}$ waves in the isolated retina begin in and propagate through Müller cells.
$\boldsymbol{A}$, Spontaneous waves are initiated from multiple points on the retina. This pseudocolor ratio image collapses $60 \mathrm{~s}$ of video by a wave propagates across the soma of the astrocyte, $\left[\mathrm{Ca}^{2+}\right]_{i}$ did not rise in the cell. $\boldsymbol{D}$, A maximum $\Delta F / F$ projection of the frames as $\boldsymbol{B})$, revealing cells in which there is a $\left[\mathrm{Ca}^{2+}\right]_{i}$ increase.

ted into the chamber. A gas collar was used as described for luciferase experiments.

High light exposure. In the animal care facilities, rats were normally exposed to $\sim 30$ lux ambient light. (Light measurements were taken in the darkest part of cages where the rats huddled.) For high light exposure experiments, rats were housed in ambient lighting of 1000 lux, beginning at $20 \mathrm{~d}$ of age. Rats were exposed to these high light conditions for 1-4 months. Both normal and high light exposure rats were on a $12 \mathrm{~h}$ light/ $12 \mathrm{~h}$ dark cycle.

Mechanical stimulus. To artificially evoke $\mathrm{Ca}^{2+}$ waves, a glass pipette was positioned with its tip touching an astrocyte. The pipette, under control of a piezoelectric actuator (PZ-150 M, Burleigh), was advanced and retracted 3-4 $\mu \mathrm{m}$ in $100 \mathrm{~ms}$. This mechanical step always triggered a large $\mathrm{Ca}^{2+}$ wave. Waves could be evoked repeatedly at a single location following a refractory period of 1-2 min, demonstrating that the mechanical stimulus did not injure the stimulated astrocyte.

Open-globe in vivo preparation. Rats were anesthetized by IP injection of urethane $(1.3 \mathrm{~g} / \mathrm{kg}$ initially, plus increments of $0.2 \mathrm{~g} / \mathrm{kg}$ as needed to reach deep anesthesia). Core body temperature was monitored and maintained at 37 degrees and hydration maintained by subcutaneous injection of saline. Animals were placed in a modified stereotaxic holder with a three-point head restraint. The right eye was secured to a metal ring by eight sutures passing through the connective tissue surrounding the globe. The cornea was removed by making an "X" cut with micro iris scissors and then cutting around the circumference of the cornea. The lens was removed by suction applied through a hypodermic needle after the anterior lens capsule was cut around its circumference. The posterior lens capsule was removed by tearing with forceps and cutting with iris scissors. The vitreous humor was removed by tearing with forceps and suction applied through the tip of a Pipetteman.

Glial cells were labeled with the $\mathrm{Ca}^{2+}$ indicator dye Oregon Green 488 BAPTA-1 (OGB-1). OGB-1 rather than Fluo-4 was used to label glial cells in vivo due to its greater $\mathrm{Ca}^{2+}$ affinity, which yielded images with a higher signal-to-noise ratio. The OGB-1 solution $(140 \mu \mathrm{g} / \mathrm{ml}$ Oregon Green, 7 $\mathrm{mg} / \mathrm{ml}$ pluronic acid and $8 \mu \mathrm{M}$ eserine in saline) was pipetted into the 


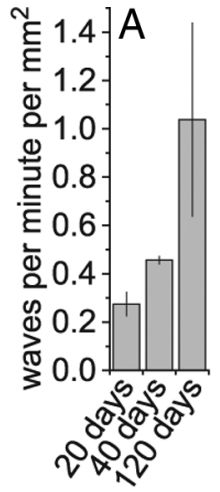

B
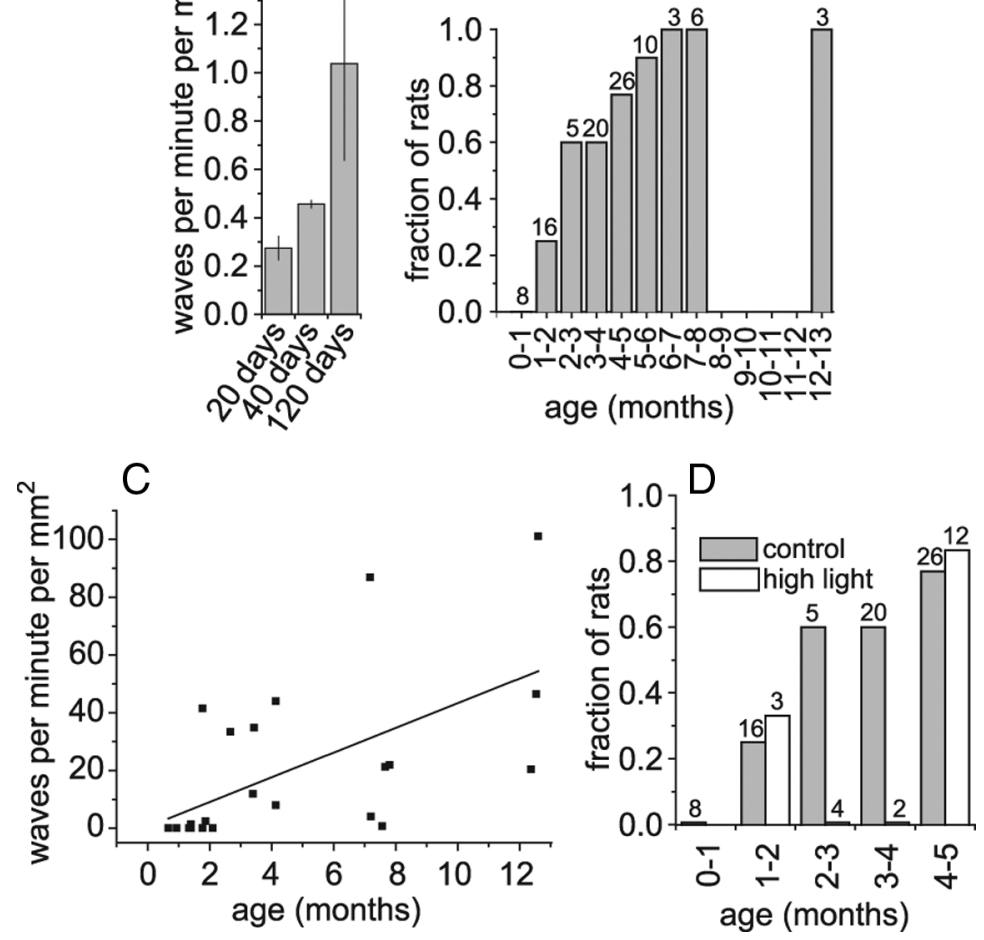

Figure 2. The frequency of spontaneous $\mathrm{Ca}^{2+}$ wave generation increases with age. $A$, The average frequency of spontaneous waves (counted from 20 to $70 \mathrm{~min}$ after the start of imaging) in animals of different ages. The frequency increases with age ( $p<$ 0.05). $\boldsymbol{B}$, The fraction of animals at each age that exhibited a high initial frequency of waves (defined as 3 or more waves occurring within the first $6 \mathrm{~min}$ of imaging). Numbers above the bars indicate the total number of animals in each bin. $C$, The highest wave rate observed in a rat increased with the rat's age $\left(p<0.005 ; R^{2}=0.33\right)$. Each point represents one animal. $\boldsymbol{D}$, Exposure to high light levels for 1-4 months did not increase the fraction of rats displaying a high initial wave frequency.

open globe following removal of the vitreous humor. Eserine (an esterase inhibitor) enhanced OGB-1 labeling of glial cells. After a 70 min incubation period, the open globe was rinsed with saline and then filled with a viscous saline solution. The stereotaxic holder and rat were fixed to a movable stage of an upright confocal microscope (Swept Field Confocal, Prairie Technologies) with the open globe facing upward. The retina was viewed through a $20 \times$ long working distance water-immersion objective (Olympus). To count spontaneous waves, the best-labeled areas of the retina were imaged for 25-110 min. Fluorescence signals were counted as waves only if (1) their shape was approximately circular, (2) they expanded concentrically over at least two frames, and (3) their peak diameter was between 30 and $100 \mu \mathrm{m}$.

Solutions and drugs. Bicarbonate-buffered Ringer's solution used for isolated retina experiments contained (in $\mathrm{mM}$ ): $111 \mathrm{NaCl}, 3 \mathrm{KCl}, 2 \mathrm{CaCl}_{2}$, $1 \mathrm{MgSO}_{4}, 0.5 \mathrm{NaH}_{2} \mathrm{PO}_{4}, 15$ dextrose, $32 \mathrm{NaHCO}_{3}$. The solution was bubbled with $95 \% \mathrm{O}_{2}, 5 \% \mathrm{CO}_{2}$. The saline solution used for in vivo experiments contained (mM): $132.5 \mathrm{NaCl}, 3 \mathrm{KCl}, 2 \mathrm{CaCl}_{2}, 1 \mathrm{MgSO}_{4}, 0.5$ $\mathrm{NaH}_{2} \mathrm{PO}_{4}, 10$ HEPES; pH 7.4. Viscous saline was made by addition of hyaluronic acid, $\mathrm{Na}$ salt $(15 \mathrm{mg} / \mathrm{ml})$ to the saline solution. All reagents were purchased from Sigma except U-46619 (Cayman), TTX (Alomone Labs), NMDA, and DL-AP7 (Tocris Bioscience), and Fluo-4, OGB-1 and pluronic acid (Invitrogen).

Statistics. Numerical values are given as mean \pm SEM, and error bars in graphs represent SEM. The single-tailed Student's $t$ test was used when comparing the means of two groups. ANOVA on a linear regression model was used when comparing the slope of the linear model to zero.

\section{Results}

Two types of macroglial cells are present in the mammalian retina: astrocytes and Müller cells. Astrocyte somata lie on the vitreal surface of the retina and extend processes that contact blood vessels in the innermost vascular layer. Müller cells are radial glia whose somata lie in the inner nuclear layer. Müller cell processes span the entire thickness of the retina and make extensive contacts with blood vessels and synapses (Newman and Reichenbach, 1996). Müller cell endfeet line the vitreal surface of the retina. Astrocytes are coupled to one another and to Müller cells by gap junctions (Zahs and Newman, 1997). Mechanical, electrical, or chemical stimulation of the inner retina evokes waves of $\mathrm{Ca}^{2+}$ elevation that propagate between both types of glial cells (Newman and Zahs, 1997; Newman, 2001).

\section{Spontaneous glial $\mathrm{Ca}^{2+}$ waves occur in the retina}

Glial $\mathrm{Ca}^{2+}$ was monitored with confocal microscopy at the inner surface of the isolated retina, where $\mathrm{Ca}^{2+}$ signals were imaged in Müller cell endfeet and astrocyte somata and processes. Glia exhibited spontaneous (i.e., unstimulated) waves of $\mathrm{Ca}^{2+}$ elevation that propagated between cells (Fig. 1A; supplemental Movie S1, available at www.jneurosci.org as supplemental material). The waves followed a pattern of concentric expansion similar to evoked waves, expanding for 4-8 s from a central point of initiation and then contracting over the next 5-10 s.

Spontaneous $\mathrm{Ca}^{2+}$ waves propagated exclusively through Müller cells and were not observed to propagate into astrocytes. In 15 waves recorded at high magnification, the waves were always initiated in Müller cells and propagated past astrocytes without a $\mathrm{Ca}^{2+}$ elevation occurring in the astrocyte ( $n=8$ waves) (Fig. $1 B-D$ ). However, we cannot rule out the possibility that astrocytes occasionally also experience $\mathrm{Ca}^{2+}$ increases. Waves propagated at $24.3 \pm 1.7 \mu \mathrm{m} / \mathrm{s}$ and reached a maximal diameter of $68.4 \pm 2.2 \mu \mathrm{m}$ ( $n=18$ waves from 4 animals $)$.

\section{Spontaneous waves increase with age}

The frequency of spontaneous wave generation was dependent on the age of the animal (Fig. $2 A$ ). The frequency equaled 1.0 per minute per $\mathrm{mm}^{2}$ in 120-d-old animals, 3.8 times higher than the 0.27 per minute per $\mathrm{mm}^{2}$ value in 20 - $\mathrm{d}$-old animals. These measurements of wave frequency were made after the retina had been in the superfusion chamber for 20-70 $\mathrm{min}$.

Some retinal areas from older animals exhibited a very high frequency of spontaneous wave generation immediately after the retinas were placed in the superfusion chamber. This high frequency of wave generation decayed within $\sim 15$ min (see Fig. $4 A$ ). A stable baseline rate of wave generation was reached after $15 \mathrm{~min}$ and was similar to the rate seen in areas of the retina where a high frequency of wave generation did not occur. The probability of retinas displaying an initial high rate of wave generation increased with the age of the animal (Fig. $2 B$ ). The maximal wave rate, measured in the first minute of recording, also increased as a function of the animal's age (Fig. $2 C$ ). In retinas from 12-monthold animals, the rate was $56 \pm 24$ waves per minute per $\mathrm{mm}^{2}$.

Because the rate of glial $\mathrm{Ca}^{2+}$ wave generation increased with age, we speculated that wave occurrence could be a consequence 

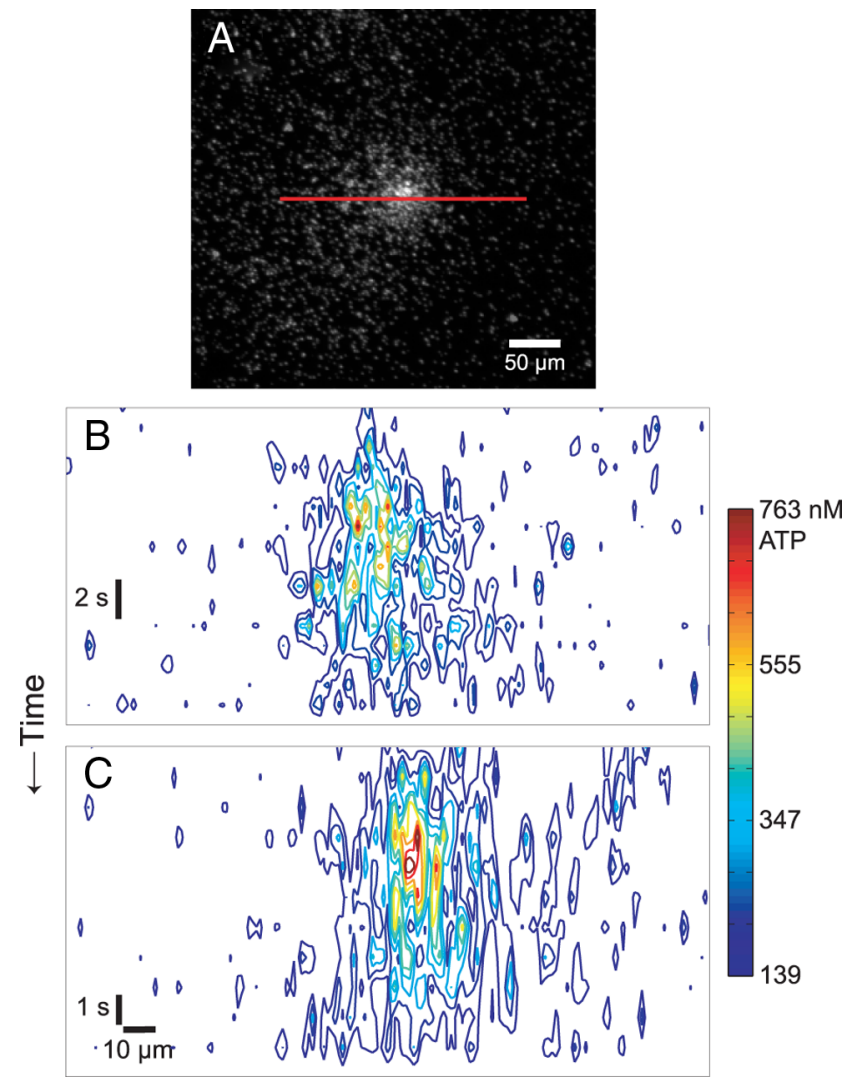

Figure 3. Spontaneous waves of ATP release in the isolated retina. ATP release was visualized with luciferin-luciferase chemiluminescence in areas of the retina with a high frequency of spontaneous $\mathrm{Ca}^{2+}$ waves. $\boldsymbol{A}$, A chemiluminescence image in $x, y$ coordinates, showing a spontaneous ATP wave at its peak. This image depicts the same spontaneous wave shown in $\boldsymbol{B} . \boldsymbol{B}, \boldsymbol{C}$, Linescan images of chemiluminescence represented as contour plots of brightness with time shown on the vertical axis. In these two examples, the linescans (illustrated by the red line in $\boldsymbol{A}$ ) are centered at the middle of each wave. Like spontaneous $\mathrm{Ca}^{2+}$ waves, the ATP waves expand from a central point (at the center/top of each graph) and then shrink, over a total period of 8-15 s.

of cumulative damage, perhaps caused by exposure to light. To test this, we exposed rats to 1000 lux ambient light $(\sim 30$ times normal light levels) for a period of 1-4 months, hypothesizing that this would hasten the development of waves. We found no effect of light treatment on the incidence of waves, however, indicating that the generation of spontaneous $\mathrm{Ca}^{2+}$ waves was not due to retinal damage caused by light exposure (Fig. 2 D). In fact, there was a trend toward reduced wave generation in lightexposed animals, but the interaction between light exposure and age was not significant by two-way ANOVA $(p=0.07)$.

\section{Spontaneous waves are propagated by release of ATP}

Artificially evoked glial $\mathrm{Ca}^{2+}$ waves in the retina are propagated from cell to cell through two mechanisms. Astrocyte-to-astrocyte propagation is mediated by diffusion of a small molecule (probably $\mathrm{IP}_{3}$; Venance et al., 1997) through gap junctions, while astrocyte-to-Müller cell and Müller cell-to-Müller cell propagation are mediated by release of ATP and its action on P2 purinergic receptors (Newman, 2001).

We asked whether the propagation of spontaneous waves through Müller cells was also mediated by ATP release. The luciferin-luciferase ATP chemiluminescence assay was used to monitor ATP release into the extracellular space during spontaneous $\mathrm{Ca}^{2+}$ wave generation. Calcium imaging was first used to
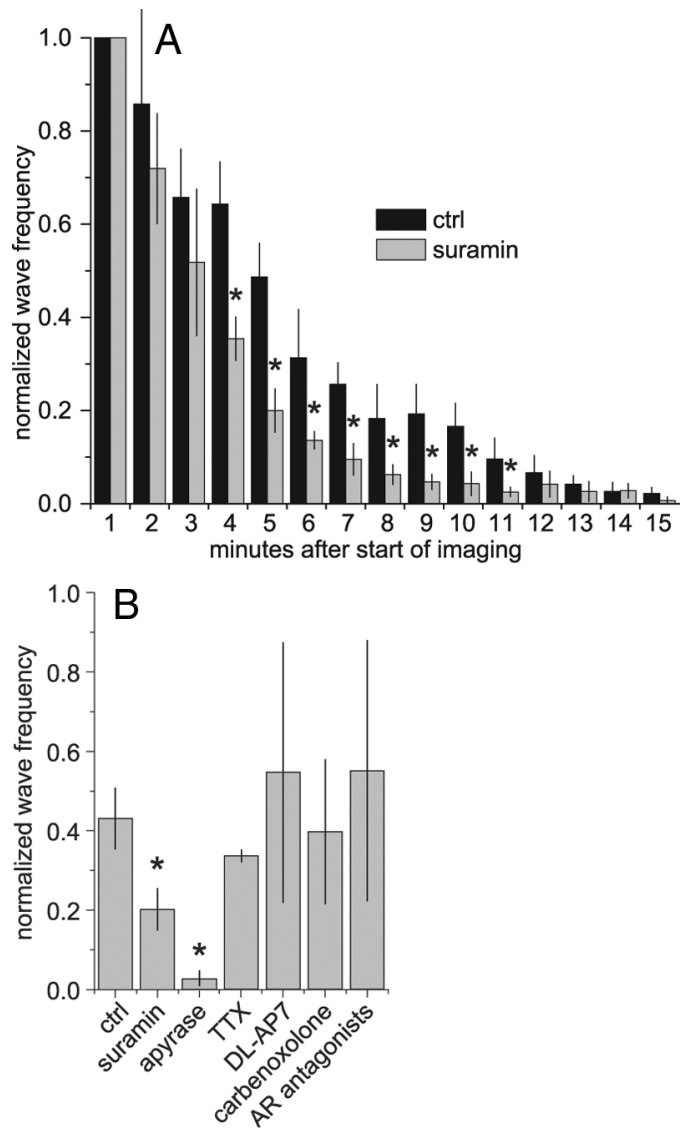

Figure 4. Spontaneous $\mathrm{Ca}^{2+}$ wave generation is dependent on $\mathrm{P} 2$ receptors and extracellular ATP. A, Suramin (100 $\mu \mathrm{m}$; P2R antagonist) decreases the frequency of spontaneous waves. The difference between control (ctrl) and suramin values is superimposed on the decay of the high initial rate of wave generation. Spontaneous $\mathrm{Ca}^{2+}$ wave counts are binned in $1 \mathrm{~min}$ intervals, and each trial is normalized to the wave count during the first minute of the trial. $\boldsymbol{B}$, Suramin (100 $\mu \mathrm{M})$ and apyrase (100 U/ml; ATP-degrading enzyme), but not TTX (200 nM), DL-AP7 (100 $\mu \mathrm{m}$; NMDAR antagonist), carbenoxolone (100 $\mu \mathrm{m}$; gap junction blocker), or a mixture of adenosine receptor antagonists (100 nm DPCPX, $1 \mu \mathrm{m}$ MRS 1191, $1 \mu \mathrm{m}$ MRS 1706, 1 $\mu \mathrm{M} Z \mathrm{ZM} 241385)$ reduced the frequency of spontaneous wave generation. Spontaneous wave counts were averaged from 3 to 6 min after the start of imaging and were normalized to wave counts during the first minute. Drugs were added 1 min after the start of imaging. ${ }^{*} p<0.05$.

locate a wave-rich region of an isolated retina from an older rat (175-325 d). ATP chemiluminescence was then imaged from the same wave-rich retinal region for several minutes. We observed multiple spontaneous chemiluminescence signals that spread outwards, indicating spontaneous waves of ATP release (Fig. 3). The propagation velocity of the spontaneous ATP waves (22.0 \pm $2.1 \mu \mathrm{m} / \mathrm{s} ; n=4$ waves) closely matched the propagation velocity of spontaneous $\mathrm{Ca}^{2+}$ waves. The maximal diameter of the ATP waves $(85.3 \pm 3.1 \mu \mathrm{m})$ was somewhat larger than that of the $\mathrm{Ca}^{2+}$ waves. The luciferin-luciferase chemiluminescence images were calibrated to determine the absolute concentration of ATP at the retinal surface. Peak extracellular ATP concentration equaled $2 \mu \mathrm{M}$ at the center of spontaneous waves of ATP release. This is an order of magnitude smaller than the peak ATP levels generated by mechanically evoked waves (Newman, 2001). This is reasonable, as the mechanically evoked waves were substantially greater in magnitude, having significantly larger diameters.

The luciferin-luciferase chemiluminescence results demonstrate that ATP is released in a spatial pattern matching the spontaneous $\mathrm{Ca}^{2+}$ waves. To test whether ATP plays a causal role in wave propagation, we blocked the action of ATP on glial puriner- 

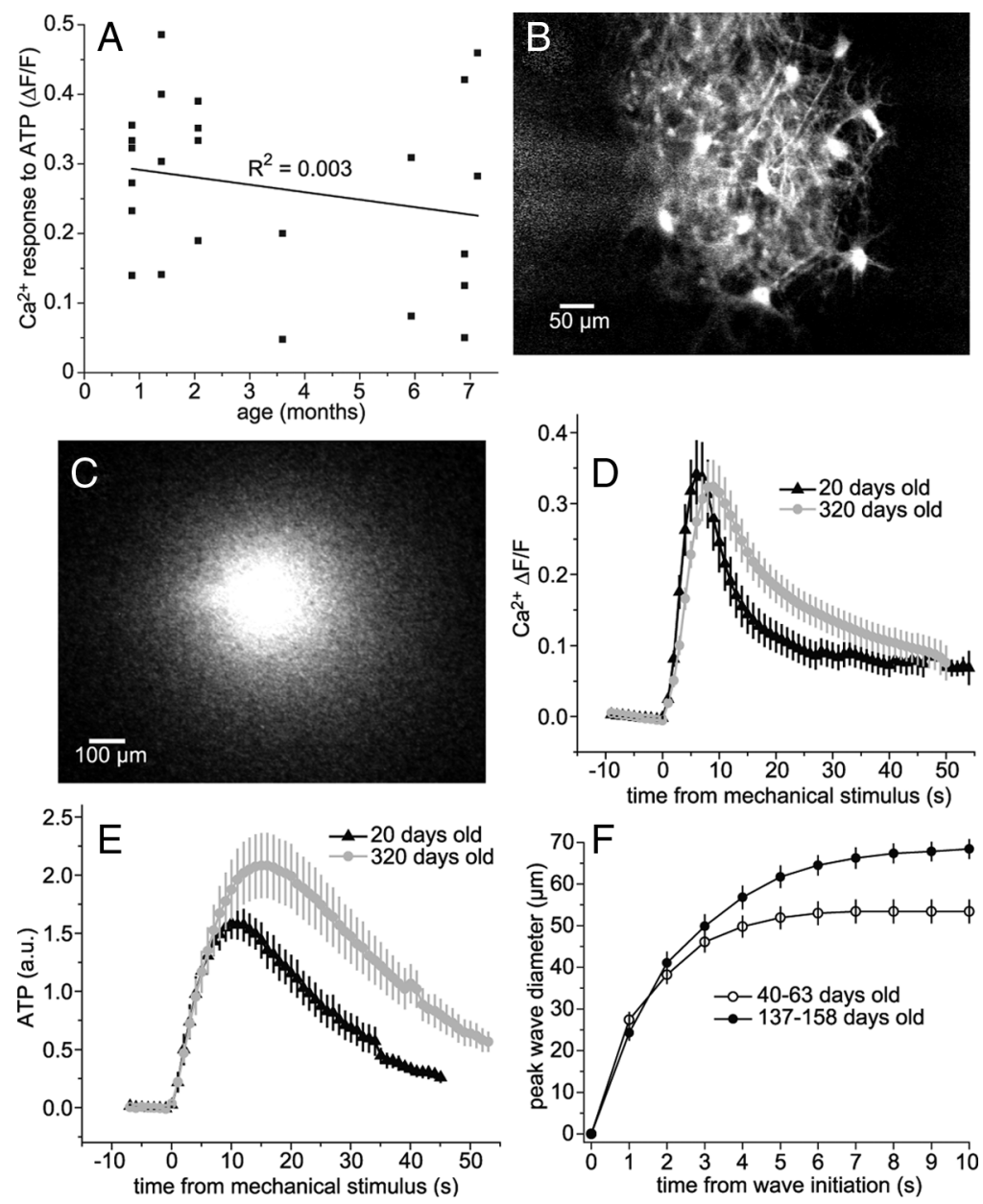

Figure 5. ATP release, but not ATP sensitivity, increases with age. A, ATP sensitivity of glial cells is age independent. Bathapplication of $3 \mu \mathrm{m}$ ATP produced a $\mathrm{Ca}^{2+}$ increase in Müller cells and astrocytes that was not correlated to the age of the animal. $\boldsymbol{B}-\boldsymbol{E}$, Mechanical stimulation of astrocyte somata triggered waves of $\mathrm{Ca}^{2+}$ and ATP. The amount of ATP released during evoked waves was greater in older animals. $\boldsymbol{B}$, An evoked $\mathrm{Ca}^{2+}$ wave (maximum brightness projection). $\boldsymbol{C}$, An evoked ATP wave (single frame at peak diameter). $\boldsymbol{D}$, The peak $\mathrm{Ca}^{2+}$ increase during evoked waves in old rats is not different from that in young rats. $\boldsymbol{E}$, The peak ATP concentration during evoked waves in old rats is higher than in young rats. Differences are significant $(p<0.05)$ from 23 to $34 \mathrm{~s}$. In $\boldsymbol{D}$ and $\boldsymbol{E}$, brightness measurements for $\mathrm{Ca}^{2+}$ fluorescence and ATP chemiluminescence are averages over the entire microscopic field. $\boldsymbol{F}$, Spontaneous $\mathrm{Ca}^{2+}$ waves reach a larger maximal diameter in older animals. The diameter shown at each time point is the maximum diameter reached by that time. Differences between younger and older rats are significant when analyzed per wave $(p<0.001)$ or per animal $(p<0.005)$.

gic receptors. Pharmacology experiments were performed during the period of high initial wave frequency in older rats (116-327 d). Suramin (a P2 receptor antagonist; $100 \mu \mathrm{M}$ ) reduced the frequency of spontaneous wave generation by $52 \pm 7 \%(n=7$ retinas) (Fig. $4 A, B$ ). Suramin has nonselective effects and might be blocking $\mathrm{Ca}^{2+}$ waves by a mechanism other than antagonizing P2 receptors. We therefore blocked the action of ATP with apyrase, an enzyme that selectively hydrolyzes ATP in the extracellular space. Apyrase $(100 \mathrm{U} / \mathrm{ml})$ reduced the frequency of spontaneous waves by $95 \pm 4 \%(n=4$ retinas) (Fig. $4 B)$. The greater effect of apyrase compared with suramin may reflect an incomplete block of $\mathrm{P} 2$ receptors by suramin.

We also tested whether gap junctions participate in spontaneous wave propagation by applying carbenoxolone, a gap junction blocker. Carbenoxolone $(100 \mu \mathrm{M})$ did not affect the number of spontaneous waves generated ( $n=3$ retinas) (Fig. $4 B$ ) or the average speed or diameter of waves (data not shown), indicating that gap junctions do not play a significant role in the propagation of spontaneous waves. This is consistent with the lack of astrocyte involvement in spontaneous waves, as wave propagation between retinal astrocytes is mediated by gap junctions but propagation between Müller cells is not (Newman, 2001). We also applied TTX to determine whether neuronal spiking contributes to the generation of spontaneous waves. TTX $(200 \mathrm{~nm})$ did not affect the number of spontaneous waves generated ( $n=4$ retinas) (Fig. $4 B$ ). In addition, neither DL-AP7 ( $n=5$ retinas) (100 $\mu \mathrm{M}$; an NMDA receptor antagonist) nor a mixture of adenosine receptor antagonists ( $n=4$ retinas) (100 nM DPCPX for A1, $1 \mu \mathrm{M}$ ZM 241385 for $\mathrm{A}_{\mathrm{A}}, 1 \mu \mathrm{M}$ MRS 1706 for $A 2_{B}$, and $1 \mu \mathrm{M}$ MRS 1191 for $\mathrm{A} 3$ receptors) had any effect on the number of spontaneous waves generated (Fig. 4B).

The pharmacology experiments illustrated in Figure 4 were conducted during the initial period of high-frequency wave generation following dissection. We also characterized $\mathrm{Ca}^{2+}$ wave generation during the period of steady, baseline generation, 20-70 min after dissection, to determine whether these waves were also propagated by ATP release. In control experiments on older animals (265-294 d), $\mathrm{Ca}^{2+}$ waves were generated at a rate of $0.74 \pm 0.43$ waves per minute per $\mathrm{mm}^{2}$ ( $n=3$ animals). Addition of apyrase (100 $\mathrm{U} / \mathrm{ml}$ ) abolished wave generation completely ( $n=3$ animals); no waves were seen during a total of $35 \mathrm{~min}$ of observation.

\section{ATP sensitivity does not increase with age}

We speculated that an increase in glial ATP sensitivity with age could account for the increase in wave generation frequency observed in older animals. To test this hypothesis, we bath-applied $3 \mu \mathrm{M}$ ATP to retinas from animals of different ages. The application of ATP always produced a $\mathrm{Ca}^{2+}$ increase in Müller cells and astrocytes, but we found no correlation between the magnitude of this increase and the age of the animal $(n=24$ animals) (Fig. 5A). To ensure that the ATP response was not saturated, we also tested ATP at $1 \mu \mathrm{M}$ and $0.3 \mu \mathrm{M}$. While the $\mathrm{Ca}^{2+}$ responses were smaller, we saw no effect of age on the magnitude of responses at these concentrations (data not shown). The results demonstrate that a change in glial cell ATP sensitivity does not underlie the increased rate of spontaneous wave generation in older animals.

\section{More ATP is released by glial cells in older animals}

Calcium wave propagation involves both ATP release and a $\mathrm{Ca}^{2+}$ elevation in response to the released ATP. Since the $\mathrm{Ca}^{2+}$ elevation observed in response to ATP does not increase with age (Fig. $5 A$ ), we speculated that the amount of ATP released during $\mathrm{Ca}^{2+}$ wave propagation might increase with age. We tested this by evoking $\mathrm{Ca}^{2+}$ waves with a mechanical stimulus in retinas from either 20-d-old or 320-d-old animals. We alternated trials be- 

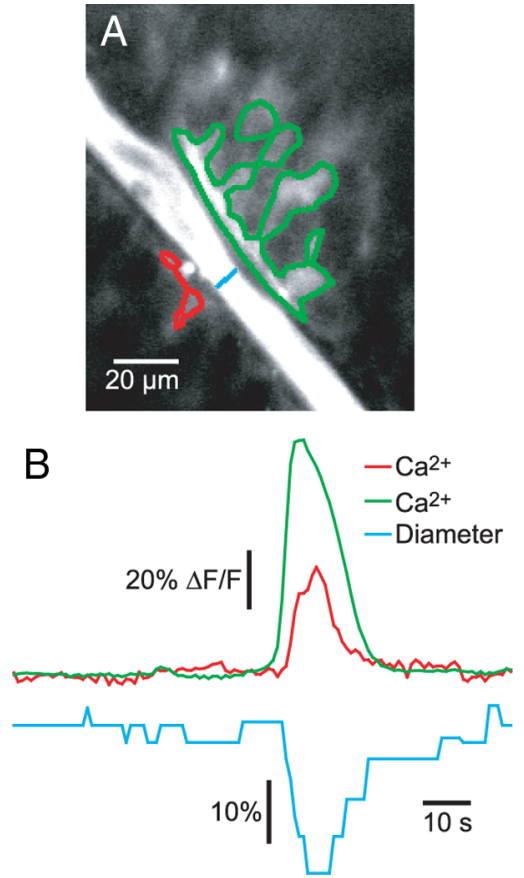

Figure 6. Spontaneous $\mathrm{Ca}^{2+}$ waves cause constrictions in arterioles. $A$, A confocal fluorescence image showing a spontaneous $\mathrm{Ca}^{2+}$ wave (maximum projection image). Glial cells are labeled with Fluo-4 and the arteriole filled with dextran-fluorescein to visualize the lumen. $B$, The arteriole constricts in synchrony with the spontaneous $\mathrm{Ca}^{2+}$ wave. The large $\mathrm{Ca}^{2+}$ rise in both red and green regions represents a spontaneous wave propagating across the vessel. The $\mathrm{Ca}^{2+}$ signals were measured in the two regions outlined in $\boldsymbol{A}$ and the vessel diameter measured at the blue line in $\boldsymbol{A}$.

tween imaging glial $\mathrm{Ca}^{2+}$ with Fluo-4 (Fig. 5B) and imaging ATP release with luciferin-luciferase (Fig. $5 C$ ). Although the peak $\mathrm{Ca}^{2+}$ increase of evoked waves was unchanged from 20 to $320 \mathrm{~d}$ (Fig. 5D), the peak ATP concentration generated by evoked waves was significantly higher in older animals than in younger animals (Fig. 5E). In intercellular glial $\mathrm{Ca}^{2+}$ waves, ATP is released in a regenerative manner (Newman, 2001). It is likely that the difference in ATP signal between older and younger animals represents an increase in regenerative ATP release, because the difference grows from 5 to $20 \mathrm{~s}$ after stimulation (Fig. $5 E$ ). Consistent with this greater ATP release in older animals, we also found that spontaneous waves reach a maximal diameter in older animals (137-158 d; $n=18$ waves) that is $28 \pm 4 \%$ larger than in young animals ( $40-63 \mathrm{~d} ; n=21$ waves) (Fig. $5 F$ ).

\section{Spontaneous waves cause blood vessels to constrict}

Artificially evoked glial $\mathrm{Ca}^{2+}$ waves, as well as $\mathrm{Ca}^{2+}$ increases evoked in single glial cells by photolysis of caged compounds, can dilate and constrict blood vessels in the retina (Metea and Newman, 2006). We investigated whether spontaneously generated $\mathrm{Ca}^{2+}$ waves have a similar effect on retinal vessels. Blood vessels and glial $\mathrm{Ca}^{2+}$ were monitored simultaneously in retinas from older animals (139-224 d) exhibiting a high frequency of $\mathrm{Ca}^{2+}$ wave generation. In three of three trials (each from a different animal) where a spontaneous wave propagated from one side of an arteriole to the other, the vessel exhibited a constriction that was synchronized with the wave (Fig. 6, supplemental Movie S2, available at www.jneurosci.org as supplemental material). In each case, the $\mathrm{Ca}^{2+}$ wave started at least $20 \mu \mathrm{m}$ from the vessel and at least two seconds before the constriction began. The constriction always began on the same video frame in which the $\mathrm{Ca}^{2+}$ wave crossed the vessel. Vessels constricted 24, 11, and 9\% of baseline diameter. These constrictions occurred over a small length of the vessel within the area of the spontaneous wave and lasted only as long as the glial $\mathrm{Ca}^{2+}$ elevation. Calcium waves which approached an arteriole but did not propagate past the vessel did not trigger vasoconstriction ( $n=41$ waves from 7 animals). Vasodilations were not observed, as expected since our Ringer's was bubbled with 95\% oxygen, which favors vessel constriction over dilation (Gordon et al., 2008; Mishra et al., 2008).

\section{Spontaneous waves occur in vivo}

Spontaneous $\mathrm{Ca}^{2+}$ waves could be an artifact of the isolated retina preparation and might not occur in vivo. We tested this by looking for spontaneous waves in retinas in vivo. We used an open-globe in vivo preparation where glial $\mathrm{Ca}^{2+}$ could be monitored in Müller cells and astrocytes with confocal microscopy. In the absence of stimulation, we observed waves of $\mathrm{Ca}^{2+}$ elevation in glia propagating outwards from a central point of initiation (Fig. 7, supplemental Movie S3, available at www.jneurosci.org as supplemental material). Waves in vivo displayed more variability in size and rate of expansion than they did in the isolated retina. However, on average, the waves expanded at $22.1 \pm 5.0 \mu \mathrm{m} / \mathrm{s}$ and achieved a maximal diameter of $67.7 \pm 9.3 \mu \mathrm{m}$ ( $n=3$ waves), very similar to the values measured in the isolated retina.

In rats $24-53 \mathrm{~d}$ old ( $n=3$ animals), we observed an average of 0.6 spontaneous waves per minute per $\mathrm{mm}^{2}$. In older rats, $84-$ $270 \mathrm{~d}$ old ( $n=4$ animals), we observed an average of 6.5 spontaneous waves per minute per $\mathrm{mm}^{2}$. Thus, there is a trend toward an increase in the frequency of spontaneous $\mathrm{Ca}^{2+}$ wave generation with age in the in vivo retina, although this effect did not reach significance. Interpolating between these values, the rate of wave generation in 120-d-old rats would be $\sim 1.8$ waves per minute per $\mathrm{mm}^{2}$, similar to the baseline wave generation rate of 1.0 per minute per $\mathrm{mm}^{2}$ measured in the isolated retina of 120-d-old rats.

\section{Discussion}

Intercellular glial $\mathrm{Ca}^{2+}$ waves constitute a coordinated signaling mechanism between glial cells and can, potentially, modulate neuronal excitability and blood flow. Recently, spontaneous waves have been observed in vivo in the cerebellum (Hoogland et al., 2009) and the cortex (Kuchibhotla et al., 2009), demonstrating that glial $\mathrm{Ca}^{2+}$ waves are a physiologically relevant phenomenon and not just a product of artificial stimulation. We report here that spontaneous waves, propagated by the release of ATP, occur in both the isolated retina and the retina in vivo.

The spontaneous retinal $\mathrm{Ca}^{2+}$ waves resemble the spontaneous waves reported in vivo in the cerebellum and cortex (Hoogland et al., 2009; Kuchibhotla et al., 2009) in some respects but differ in others. Whereas the waves in cortex were seen only near amyloid plaques in Alzheimer's mice (and did not occur in wild-type animals), waves in retina and cerebellum occurred in the absence of apparent pathology. The waves in cortex, although expanding at a rate similar to waves in retina, reached a maximum diameter of $\sim 200 \mu \mathrm{m}$, similar to waves seen in culture, but much larger than the spontaneous waves observed in cerebellum and in the retina. The rate of spontaneous wave generation was not reported in cerebellum or cortex.

Like the artificially evoked $\mathrm{Ca}^{2+}$ waves reported in the isolated retina (Newman and Zahs, 1997) and in brain slices (Dani et al., 1992), spontaneous waves in the retina are mediated by release of ATP and activation of glial purinergic receptors. ATP imaging reveals spontaneous waves of ATP release matching the sponta- 

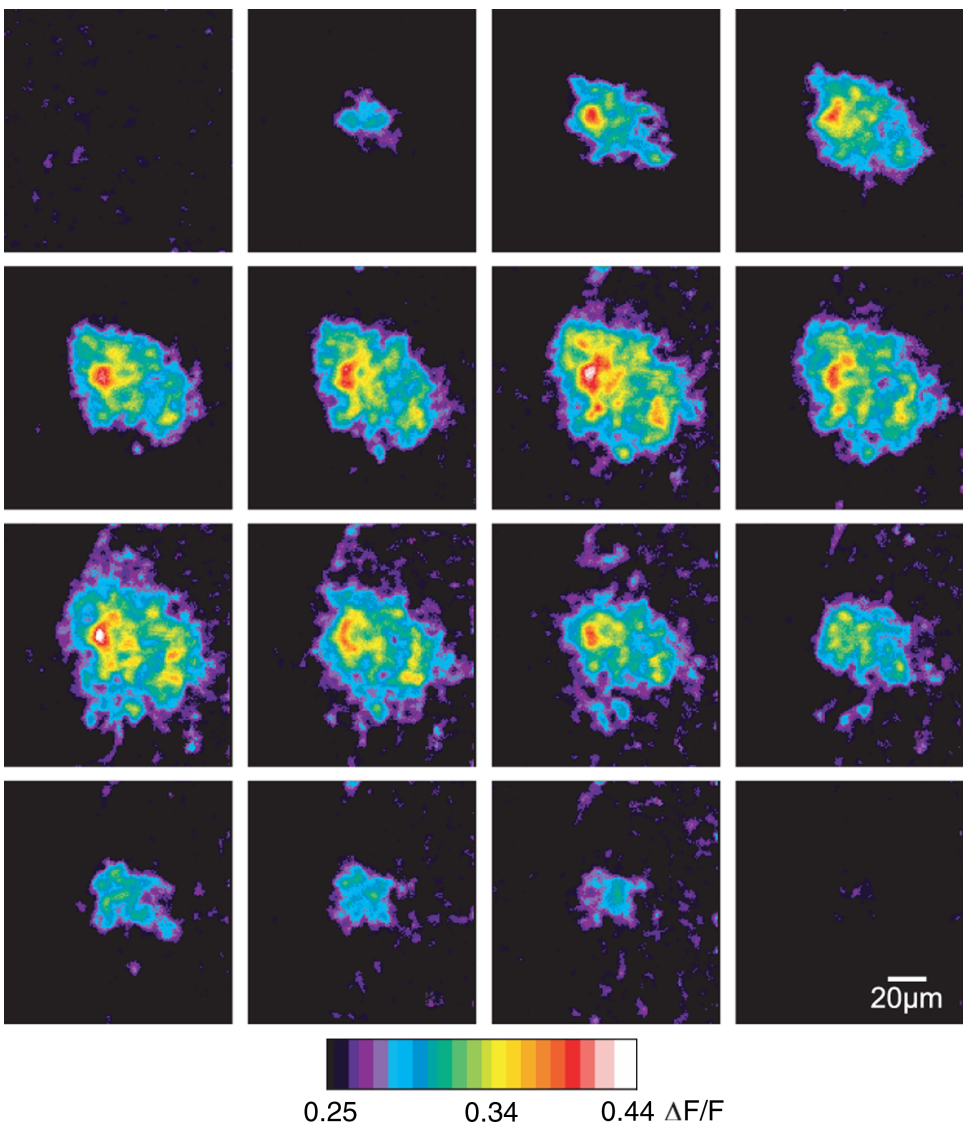

Figure 7. Spontaneous $\mathrm{Ca}^{2+}$ waves occur in the retina in vivo. These pseudocolor $\Delta F / F$ ratio images show a time series (left to right, top to bottom) of a single spontaneous $\mathrm{Ca}^{2+}$ wave in vivo. Each frame represents $1 \mathrm{~s}$. The focal plane is a few micrometers beneath the surface of the retina, in the ganglion cell layer, where the brightest Müller cell $\mathrm{Ca}^{2+}$ signals are observed.

neous $\mathrm{Ca}^{2+}$ waves (Fig. 3). Both suramin, a purinergic antagonist, and apyrase, an ATP-degrading enzyme, reduce the frequency of spontaneous waves in the retina. Neither gap junctional coupling between glial cells nor neuronal activity play a significant role in the generation and propagation of spontaneous waves. Spontaneous $\mathrm{Ca}^{2+}$ waves observed in vivo in the cerebellum are also mediated by activation of purinergic receptors (Hoogland et al., 2009).

Spontaneous $\mathrm{Ca}^{2+}$ waves in the retina are initiated in and propagate through Müller cells and do not appear to involve astrocytes. Although evoked glial $\mathrm{Ca}^{2+}$ waves in the retina propagate through astrocytes if they are initiated in astrocytes, evoked waves that are initiated in Müller cells rarely propagate into astrocytes (Newman, 2001). The difference could be attributable to astrocytes having a higher threshold for $\mathrm{Ca}^{2+}$ activation by purinergic receptors than Müller cells.

Some retinas, especially from older animals, exhibited a high initial frequency of spontaneous waves that decayed to a stable baseline within $15 \mathrm{~min}$. We speculate that the high initial wave rate may be a consequence of mechanical trauma suffered during dissection. However, it is likely that the waves that occur during the initial high-frequency period are generated by the same mechanism as those observed during the stable period of lowfrequency wave generation as they have similar properties, including propagation velocity, size and sensitivity to apyrase.

The frequency of spontaneous $\mathrm{Ca}^{2+}$ wave generation increases significantly with the age of the animal. In the isolated retina, the stable, basal rate of wave generation increases from 0.27 to 1.0 waves per minute per $\mathrm{mm}^{2}$ as animals age from 20 to
120 d. A similar increase in the rate of spontaneous wave generation is seen in the retina in vivo. The increase could be related to pathology associated with aging. It is not due to damage produced by light exposure, however, as animals exposed to high ambient light for 1-4 months did not exhibit increased wave generation (Fig. 2D). ATP-evoked $\mathrm{Ca}^{2+}$ increases in retinal glial cells did not increase with age, suggesting that increased wave frequency is not due to an upregulation of $\mathrm{P} 2$ receptors or downstream signaling molecules in glial cells. However, the ATP released during evoked $\mathrm{Ca}^{2+}$ waves did increase with age, suggesting that an increase in glial ATP release could account for the age-related increase in spontaneous wave generation. In the isolated retina, individual Müller cells, but not astrocytes, frequently display transient, non-propagating $\mathrm{Ca}^{2+}$ increases (Newman, 2005). If spontaneous waves begin as single-cell $\mathrm{Ca}^{2+}$ transients, it is possible that greater ATP release in older animals in response to $\mathrm{Ca}^{2+}$ increases permits more of these transients to propagate as waves. This could account for the higher frequency of wave generation observed in older animals. Consistent with an age-dependent increase in glial ATP release, spontaneous $\mathrm{Ca}^{2+}$ waves reached a larger peak diameter in older animals (Fig. 5F).

Spontaneous glial $\mathrm{Ca}^{2+}$ waves could contribute to pathology in the CNS. Under ischemic conditions in the cortex in vivo, not only are spontaneous glial $\mathrm{Ca}^{2+}$ transients increased compared with healthy brain, but they are also spatially coordinated and contribute to neuronal death (Ding et al., 2009). In Alzheimer's mice, spontaneous $\mathrm{Ca}^{2+}$ waves occur near amyloid plaques (Kuchibhotla et al., 2009), suggesting that the generation of waves could be triggered by pathology.

Conversely, spontaneous $\mathrm{Ca}^{2+}$ waves could act to protect retinal cells. Activation of P2Y receptors on astrocytes boosts the resistance of both the astrocytes and nearby neurons to oxidative stress (Wu et al., 2007). Spontaneous waves could serve as a source of extracellular ATP to stimulate this protective effect, which would become increasingly important in older animals as oxidative stress increases (Sohal and Weindruch, 1996; Kregel and Zhang, 2007).

In addition to their possible role in pathology, spontaneous glial $\mathrm{Ca}^{2+}$ waves comprise a functional signaling pathway that may have important downstream effects on blood vessels and neurons. Artificially evoked $\mathrm{Ca}^{2+}$ waves regulate the diameter of retinal arterioles by the release of arachidonic acid metabolites (Mulligan and MacVicar, 2004; Metea and Newman, 2006). We demonstrate here that spontaneously generated $\mathrm{Ca}^{2+}$ waves can also regulate arteriole diameter, implying that spontaneous waves contribute to the regulation of blood flow. Arteriole constrictions were observed, as expected because the retina was superfused with Ringer's bubbled with 95\% oxygen. When the oxygen concentration is lowered to a physiological level, it is likely that spon- 
taneous $\mathrm{Ca}^{2+}$ waves would evoke vasodilations instead of vasoconstrictions (Gordon et al., 2008; Mishra et al., 2008).

Spontaneous waves may also influence neuronal activity. Evoked increases in glial $\mathrm{Ca}^{2+}$ lead to gliotransmitter release that can modulate neuronal excitability and synaptic transmission (Newman, 2004; Agulhon et al., 2008). ATP release from glia causes depression of synaptic transmission in cultured hippocampal neurons (Koizumi et al., 2003) and brain slices (Pascual et al., 2005). Spontaneous $\mathrm{Ca}^{2+}$ transients in individual glial cells cause NMDA receptor-mediated currents in neurons (Parri et al., 2001; Angulo et al., 2004; Fellin et al., 2004). In the retina, stimulated $\mathrm{Ca}^{2+}$ increases in glia cause neuronal hyperpolarization mediated by ATP release (Newman, 2003), and evoked waves can facilitate or depress lightevoked neuronal spiking (Newman and Zahs, 1997). In light of these functional consequences of glial $\mathrm{Ca}^{2+}$ increases, changes in wave generation over the course of early adulthood could represent agedependent changes in information processing in the retina.

\section{References}

Aguado F, Espinosa-Parrilla JF, Carmona MA, Soriano E (2002) Neuronal activity regulates correlated network properties of spontaneous calcium transients in astrocytes in situ. J Neurosci 22:9430-9444.

Agulhon C, Petravicz J, McMullen AB, Sweger EJ, Minton SK, Taves SR, Casper KB, Fiacco TA, McCarthy KD (2008) What is the role of astrocyte calcium in neurophysiology? Neuron 59:932-946.

Angulo MC, Kozlov AS, Charpak S, Audinat E (2004) Glutamate released from glial cells synchronizes neuronal activity in the hippocampus. J Neurosci 24:6920-6927.

Araque A, Sanzgiri RP, Parpura V, Haydon PG (1998) Calcium elevation in astrocytes causes an NMDA receptor-dependent increase in the frequency of miniature synaptic currents in cultured hippocampal neurons. J Neurosci 18:6822-6829.

Cornell-Bell AH, Finkbeiner SM, Cooper MS, Smith SJ (1990) Glutamate induces calcium waves in cultured astrocytes: long-range glial signaling. Science 247:470-473.

Dani JW, Chernjavsky A, Smith SJ (1992) Neuronal activity triggers calcium waves in hippocampal astrocyte networks. Neuron 8:429-440.

Ding S, Fellin T, Zhu Y, Lee SY, Auberson YP, Meaney DF, Coulter DA, Carmignoto G, Haydon PG (2007) Enhanced astrocytic $\mathrm{Ca}^{2+}$ signals contribute to neuronal excitotoxicity after status epilepticus. J Neurosci 27:10674-10684.

Ding S, Wang T, Cui W, Haydon PG (2009) Photothrombosis ischemia stimulates a sustained astrocytic $\mathrm{Ca}^{2+}$ signaling in vivo. Glia 57:767-776.

Fellin T, Carmignoto G (2004) Neurone-to-astrocyte signalling in the brain represents a distinct multifunctional unit. J Physiol 559:3-15.

Fellin T, Pascual O, Gobbo S, Pozzan T, Haydon PG, Carmignoto G (2004) Neuronal synchrony mediated by astrocytic glutamate through activation of extrasynaptic NMDA receptors. Neuron 43:729-743.

Gordon GR, Choi HB, Rungta RL, Ellis-Davies GC, MacVicar BA (2008) Brain metabolism dictates the polarity of astrocyte control over arterioles. Nature 456:745-749.

Haas B, Schipke CG, Peters O, Söhl G, Willecke K, Kettenmann H (2006) Activity-dependent ATP-waves in the mouse neocortex are independent from astrocytic calcium waves. Cereb Cortex 16:237-246.

Hirase H, Qian L, Bartho P, Buzsaki G (2004) Calcium dynamics of cortical astrocytic networks in vivo. PLoS Biol 2:494-499.

Hoogland TM, Kuhn B, Göbel W, Huang W, Nakai J, Helmchen F, Flint J, Wang SS (2009) Radially expanding transglial calcium waves in the intact cerebellum. Proc Natl Acad Sci U S A 106:3496-3501.

Kanemaru K, Okubo Y, Hirose K, Iino M (2007) Regulation of neurite growth by spontaneous $\mathrm{Ca}^{2+}$ oscillations in astrocytes. J Neurosci 27:8957-8966.

Kang J, Jiang L, Goldman SA, Nedergaard M (1998) Astrocyte-mediated potentiation of inhibitory synaptic transmission. Nat Neurosci 1:683-692.

Koizumi S, Fujishita K, Tsuda M, Shigemoto-Mogami Y, Inoue K (2003) Dynamic inhibition of excitatory synaptic transmission by astrocytederived ATP in hippocampal cultures. Proc Natl Acad Sci U S A 100:11023-11028.

Kregel KC, Zhang HJ (2007) An integrated view of oxidative stress in aging: basic mechanisms, functional effects, and pathological considerations. Am J Physiol Regul Integr Comp Physiol 292:R18-R36.

Kuchibhotla KV, Lattarulo CR, Hyman BT, Bacskai BJ (2009) Synchronous hyperactivity and intercellular calcium waves in astrocytes in Alzheimer mice. Science 323:1211-1215.

Metea MR, Newman EA (2006) Glial cells dilate and constrict blood vessels: a mechanism of neurovascular coupling. J Neurosci 26:2862-2870.

Mishra A, Hu M, Newman EA (2008) Nitric oxide and oxygen modulate glialmediated neurovascular coupling. In: Glia in health and disease (Barres B, Stevens B, eds). Cold Spring Harbor, NY: Cold Spring Harbor Laboratories.

Mulligan SJ, MacVicar BA (2004) Calcium transients in astrocyte endfeet cause cerebrovascular constrictions. Nature 431:195-199.

Nedergaard M (1994) Direct signaling from astrocytes to neurons in cultures of mammalian brain cells. Science 263:1768-1771.

Nett WJ, Oloff SH, McCarthy KD (2002) Hippocampal astrocytes in situ exhibit calcium oscillations that occur independent of neuronal activity. J Neurophysiol 87:528-537.

Newman EA (2001) Propagation of intercellular calcium waves in retinal astrocytes and Müller cells. J Neurosci 21:2215-2223.

Newman EA (2003) Glial cell inhibition of neurons by release of ATP. J Neurosci 23:1659-1666.

Newman EA (2004) Glial modulation of synaptic transmission in the retina. Glia 47:268-274.

Newman EA (2005) Calcium increases in retinal glial cells evoked by lightinduced neuronal activity. J Neurosci 25:5502-5510.

Newman E, Reichenbach A (1996) The Muller cell: a functional element of the retina. Trends Neurosci 19:307-312.

Newman EA, Zahs KR (1997) Calcium waves in retinal glial cells. Science 275:844-847.

Newman EA, Zahs KR (1998) Modulation of neuronal activity by glial cells in the retina. J Neurosci 18:4022-4028.

Parpura V, Basarsky TA, Liu F, Jeftinija K, Jeftinija S, Haydon PG (1994) Glutamate-mediated astrocyte-neuron signalling. Nature 369:744-747.

Parri HR, Gould TM, Crunelli V (2001) Sponataneous astrocytic $\mathrm{Ca}^{2+}$ oscillations in situ drive NMDAR-mediated neuronal excitation. Nat Neurosci 4:803-812.

Pascual O, Casper KB, Kubera C, Zhang J, Revilla-Sanchez R, Sul JY, Takano H, Moss SJ, McCarthy K, Haydon PG (2005) Astrocytic purinergic signaling coordinates synaptic networks. Science 310:113-116.

Porter JT, McCarthy KD (1996) Hippocampal astrocytes in situ respond to glutamate released from synaptic terminals. J Neurosci 16:5073-5081.

Scemes E, Giaume C (2006) Astrocyte calcium waves: what they are and what they do. Glia 54:716-725.

Schipke CG, Boucsein C, Ohlemeyer C, Kirchhoff F, Kettenmann H (2002) Astrocyte $\mathrm{Ca}^{2+}$ waves trigger responses in microglial cells in brain slices. FASEB J 16:255-257.

Sohal RS, Weindruch R (1996) Oxidative stress, caloric restriction, and aging. Science 273:59-63.

Stosiek C, Garaschuk O, Holthoff K, Konnerth A (2003) In vivo two-photon calcium imaging of neuronal networks. Proc Natl Acad Sci U S A 100:7319-7324.

Takano T, Tian GF, Peng W, Lou N, Libionka W, Han X, Nedergaard M (2006) Astrocyte-mediated control of cerebral blood flow. Nat Neurosci 9:260-267.

Tian GF, Takano T, Lin JH, Wang X, Bekar L, Nedergaard M (2006) Imaging of cortical astrocytes using 2-photon laser scanning microscopy in the intact mouse brain. Adv Drug Deliv Rev 58:773-787.

Venance L, Stella N, Glowinski J, Giaume C (1997) Mechanism involved in initiation and proagation of receptor-induced intercellular calcium signalling in cultured rat astrocytes. J Neurosci 17:1981-1992.

Wu J, Holstein JD, Upadhyay G, Lin DT, Conway S, Muller E, Lechleiter JD (2007) Purinergic receptor-stimulated IP3-mediated $\mathrm{Ca}^{2+}$ release enhances neuroprotection by increasing astrocyte mitochondrial metabolism during aging. J Neurosci 27:6510-6520.

Yang Y, Ge W, Chen Y, Zhang Z, Shen W, Wu C, Poo M, Duan S (2003) Contribution of astrocytes to hippocampal long-term potentiation through release of D-serine. Proc Natl Acad Sci U S A 100:15194-15199.

Zahs KR, Newman EA (1997) Asymetric gap junctional coupling between glial cells in the rat retina. Glia 20:10-22.

Zonta M, Angulo MC, Gobbo S, Rosengarten B, Hossmann KA, Pozzan T, Carmignoto G (2003) Neuron-to-astrocyte signaling is central to the dynamic control of brain microcirculation. Nat Neurosci 6:43-50. 